\title{
Supporting Drivers in Keeping Safe Speed in Adverse Weather Conditions by Mitigating the Risk Level
}

\author{
Romain Gallen, Nicolas Hautière Member, IEEE, Aurélien Cord and Sébastien Glaser
}

\begin{abstract}
Overspeeding is both a cause and an aggravation factor of traffic accidents. Consequently, lots of efforts are devoted so as to limit overspeeding and consequently to increase the safety of road networks. In this article, a novel approach to compute a safe speed profile to be used in an adaptive Intelligent Speed Adaptation system (ISA) is proposed. The method presents two main novelties. First, the $85^{t h}$ percentile of observed speeds $\left(V_{85}\right)$, estimated along a road section, is used as a reference speed, practiced and practicable in ideal conditions. Second, this reference speed is modulated in adverse weather conditions in order to account for a reduced friction and a reduced visibility distance. The risk is thus mitigated by modulating the potential severity of crashes by means of a generic scenario of accident. Within this scenario, the difference in speed that should be applied in adverse conditions is estimated so that the highway risk is the same as in ideal conditions. The system has been tested on actual data collected on a French secondary road and implemented on a test track and a fleet of vehicles. The performed tests and the experiments of acceptability show a great interest for the deployment of such a system.
\end{abstract}

Index Terms-Safe speed, visibility, friction, cooperative systems, risk.

\section{INTRODUCTION}

W ITH 1.27 millions deaths annually worldwide, road crashes are of major concern. They were the ninth source of deaths in the world and are expected to be the fifth source in 2030 in developed countries as in developing regions [1], [2]. Indeed, speed is cited as the first factor; it is considered to be the cause in one third of road crashes [3], [4] but it also impacts the severity of accidents. Many attempts at reducing road fatalities and injuries have been undertaken over the past fifty years, from speed limitations to seat belt use and drug enforcement. In the past decade, automated control of speeds has proven very efficient to reduce accidents in England, Netherlands and in France. It is estimated to have helped reduce fatality in France by 25\% between 2003 and 2007 according to [5]. But recent trends showed a stagnation, suggesting that it has reached its full potential for safety benefits.

The development of Advanced Driver Assistance Systems (ADAS) is a very active field of research in the automotive

R. Gallen is with CETMEF, Plouzané, France email: romain.gallen@developpement-durable.gouv.fr.

N. Hautière is with the Universite Paris-Est, LEPSIS, COSYS, IFSTTAR, Paris, France e-mail: nicolas.hautiere@ifsttar.fr.

A. Cord and S. Glaser are with UniverSud, LIVIC, COSYS, IFSTTAR, Versailles, France e-mail: \{aurelien.cord, sebastien.glaser\}@ifsttar.fr.

Manuscript received Month xx, 20xx; revised Month xx, 20xx. industry. Systems relying on proprioceptive sensors such as the Anti-lock Braking System (ABS) or the Electronic Stability Program (ESP) are widely integrated in today's cars. Other systems rely on exteroceptive sensors (LIDAR, RADAR, camera) such as Lane Departure Warning (LDW), Forward Collision Warning (FCW), Traffic Sign Recognition (TSR) or Advanced Front lighting Systems (AFS).

In this context, ADAS focusing on speed such as Intelligent Speed Adaptation (ISA) systems are considered as having high potential for road safety [6], whatever their types (static vs. dynamic) and modes (mandatory vs. advisory), mainly because of the drop in number of crashes due to average speed decrease, all other things being equal. This has been formalized first by Nilsson's power model between speed and accident probability [7], later reviewed in [8]. Other works like [9], [10] confirm that crash-incidence generally declines as a result of speed limit reduction. The second reason for considering ISA as beneficial is the impact of speed on crash severity. Lower speeds mechanically lead to less severe accidents.

ISA systems can work in advisory or mandatory modes. Mandatory modes have only been tested in research projects such as [11]-[13]. The safety benefits of ISAs are estimated to be higher for a dynamic ISA in that mode (-44\% of fatality) compared to the advisory mode ( $-9 \%$ of fatality) in case of full penetration of ISA according to [14]. However recent estimations of safety benefits for ISAs in France [15] have shown lesser potential, ranging from $4 \%$ to $16 \%$ depending both on mode and type of road. Current implementations of ISAs compliant with road regulation already exist in advisory mode in many cars with the use of speed limit detection algorithms using cameras such as [16].

Road design and legal speed limit are strongly linked. New roads are designed according to an iterative process between the needs and building constraints. Curvature, slope and superelevation of the road are consistent with the foreseen speed limit. Secondary roads are often ancient and have been created before recent design models were created, presenting very tight curves, sections with a low sight distance for instance. Two thirds of road fatalities in France happen on secondary roads and one third of the fatalities imply a single vehicle. Only road signs and speed limit signs are then used to inform the driver about the difficulties of the infrastructure characteristics in order to help him adapt his practiced speed. Because they are static and localized, the posted speed limits are not adaptive enough to build accurate safety measures. 
Sometimes, at local scale, the posted speed limit should be seen as a legal speed limit but should not be considered as an advisable speed, especially in adverse meteorological conditions when visibility or friction are reduced.

Among recent developments are adaptive dynamic ISAs, whose aim is to cope with various conditions, especially road curvature, lower friction, and poor visibility. Finding a safe speed recommended for a single driver in various conditions remains a challenging issue [17]. Curve Warning Systems (CWS) can adapt speed in curves if the speed limit is not suitable such as [18], [19]. The principles for the implementation of an ISA in adverse conditions have been proposed in [20], [21]. These last two methods are based on the same safety criterion: The driver must be able to stop the vehicle in the visibility distance on wet, slippery or dry roads.

In the cooperative framework of an adaptive and dynamic ISA system, this paper presents the onboard computation of a safe speed in real-time along an itinerary. On one side, contextual information on conditions such as fog [22], [23], rainy situation [24], [25] or wet road [26] is estimated in real-time by in-vehicle sensors. On the other side, roadway information is used (reference speed, curvature, slope or superelevation) in order to expand the electronic horizon before the car. This information is then fused online and the reference speed is modulated depending on environmental and geometric characteristics of the roadway. Our method is original in that the safe speed is not computed from models (bottomup approach like [21]) but instead by modulating a reference speed practiced and practicable in good conditions (top-down approach), in order to get the same risk in adverse weather conditions as in good weather conditions. The proposed safety criterion, called "Equivalent Total Risk" (ETR), takes into account the potential severity of a crash using accident statistics. It is new and less constraining as compared with the"stopping distance" or "zero risk" strategy used by [20] and [21]. The presented method is generic enough to be open and adaptable to specific vehicles, drivers or road networks if one provides the corresponding inputs to the system.

This paper is organized as follows. Section II defines the concept of highway risk as well as the materials needed to implement this definition, namely one crash severity metric and one emergency braking model. In section III, the proposed risk migitation based on the ETR criterion is presented and is applied at one point on the road for a given severity level. In section IV, the ETR strategy is applied with simulated adverse conditions along an actual road section on which the characteristics and reference speed have been collected. In section $\mathrm{V}$, the output of the system on a real path is shown and analyzed. Finally, section VI presents the conclusion and the next steps of this work.

\section{HighWAY RISK}

In this section, the concept of highway risk is defined. Then, the materials needed to implement this definition are detailed: crash severity metrics, emergency braking model. Ultimately, thanks to these different elements, the notion of risk level can be mathematically defined.

\section{A. Definition of Highway Risk}

Risk can be defined as the combination of the probability of an accident with its severity. Our risk definition is based on a basic scenario of accident. While the case vehicle is driving in free-flow conditions, an emergency situation occurs. The driver begins an emergency braking maneuver and eventually crashes against a rigid fixed obstacle. The emergency situation, whatever its cause (distraction, surprise), may happen anywhere during the trip. In this scenario, it is considered that there is an equiprobability of obstacle appearance depending on the distance. The driver may run off the road anytime after the detection of an emergency situation and then hit an obstacle. In this scenario, the driver brakes on the road and hits an obstacle on the carriageway or onthe roadside. As the friction outside the road is assumed to be negligible, the driver hits the obstacle with the same speed that he had when he ran off-road. The severity of the accident depends on the speed of the crash and of the nature of the obstacle and of the hitting configuration as defined in the next section.

\section{B. Crash Severity Metrics}

The potential severity of an accident depends on the speed of a crash as well as on its configuration (frontal collision with a rigid fixed object, with another vehicle or with a stopped vehicle). Many studies have described the link between speed of crashes and severity for the driver or for other vehicle occupants. This severity may depend on driver characteristics such as age, gender or weight [3], [27], on the vehicle safety devices [28], [29], on the direction of collision [30], on the mass ratio in two-vehicle crashes [31] or on the size of cars [32].

Many different metrics linked to speed are used to assess the potential severity of crashes. These are Kinetic Energy Equivalent Speed (KEES or EES), Equivalent Barrier Speed (EBS), Occupant Impact Velocity (OIV) or Acceleration Severity Index (ASI). In [33], the severity of an accident is described using Maximum Accident Injury Severity (MAIS) and the variation of speed during the accident for both frontal and side impacts. Some of these measures were designed to relate vehicle kinematics at the instant of crash (derived from post crash observation of vehicle deformation) while other speed related parameters were designed to study potential injury severity. They can be computed with data from post-crash analysis of vehicles or nowadays from Event Data Recorders (EDR) integrated in cars. OIV and ASI were found by [34] to offer no significant predictive advantage over the simpler delta- $\mathrm{V}(\Delta V)$. Delta- $\mathrm{V}$ is an indication of the acceleration experienced by car occupants while EES assesses the work done in crushing the car structure [35].

According to [36], from analysis of collision data for Britain, Australia and the US, the collisions can all be considered to be frontal impacts for high collision severity levels (i.e. high $\Delta V$ values). The risk for vehicle occupants is estimated by using statistics giving injury severity probability as a function of delta- $\mathrm{V}$ in frontal impacts taken from [37] as shown on Fig. 1. 


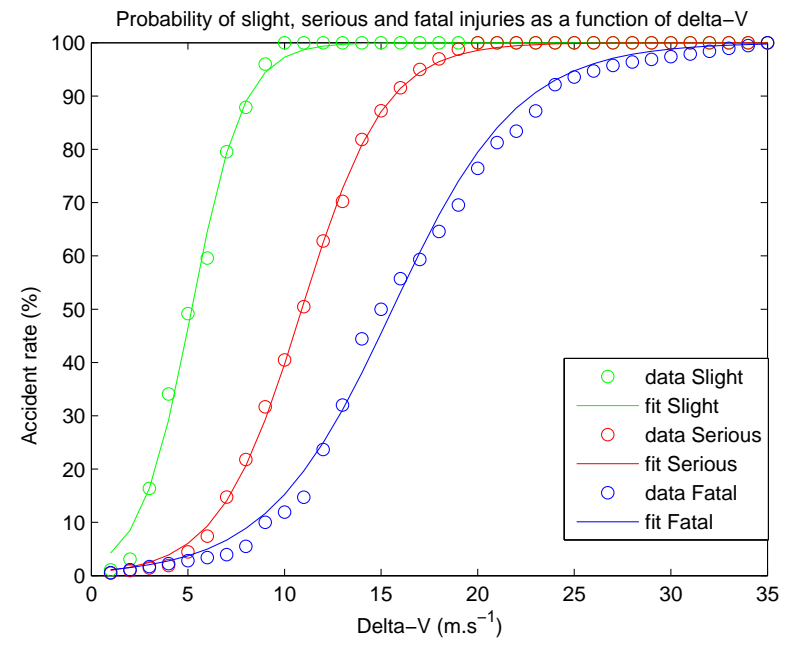

Fig. 1. Cumulative speed curves for drivers in frontal impacts, data from [37] and curve fitting.

In order to dispose of continuous cumulative crash severity curves depending on delta- $\mathrm{V}$, sigmoid functions were fitted to the data from [37]. They are of the logit form:

$$
P I_{\text {Severity }}(\Delta V)=\frac{a_{\text {Severity }}}{1+e^{-\frac{\Delta V-b_{\text {Severity }}}{c_{\text {Severity }}}}}
$$

where $a, b$ and $c$ are coefficients that vary based on the severity, which can be either slight, serious or fatal. There are three sets of coefficients given in Tab. I, one for each probability of injury (PI) curve such as presented in Fig. 1.

\section{Emergency Braking Model}

Any model of infrastructure-driver-car interactions able to compute a braking speed profile in emergency situations could be used in the framework we propose. The proposed model is simple and illustrative of our capabilities to account for infrastructure, vehicle and driver characteristics that we measured and were able to feed the system as inputs. The speed profile during an emergency braking is computed using measured local characteristics of the road such as curvature, slope angle, superelevation and friction as presented in Sec. IV-B. Driver related parameters such as reaction time and pressure on the brake pedal are also used in the model of the vehicle dynamics. Finally, vehicle related parameters such as the presence of ABS are used to compute these speed profiles.

After an emergency situation arises, the driver needs some time to be aware of the situation and to start pressing the brake

TABLE I

COEFFICIENTS OF FITTING FOR PROBABILITY OF INJURY (PI) CURVES DEPENDING ON SEVERITY AS SHOWN ON FIG. 1

\begin{tabular}{|c||c|c|c|}
\hline Severity & \multicolumn{3}{|c|}{ Coefficients } \\
\hline \hline Slight & $a_{S l}=100$ & $b_{S l}=5.19$ & $c_{S l}=1.34$ \\
Serious & $a_{S e}=100$ & $b_{S e}=10.9$ & $c_{S e}=2.15$ \\
Fatal & $a_{F a}=100$ & $b_{F a}=15.6$ & $c_{F a}=3.26$ \\
\hline
\end{tabular}

pedal. During this time of perception and reaction, the speed is kept constant and the distance $D_{\text {Reac }}$ covered is:

$$
D_{\text {Reac }}=V_{0} t_{P R}
$$

with $V_{0}$ the speed in $m . s^{-1}$ and $t_{P R}$ the perception-reaction time in seconds. Once the driver has covered this distance with constant speed, the emergency braking takes place with a starting speed equal to $V_{0}$. The emergency braking speed profiles are computed differently on straight sections and in curves.

1) Braking on straight sections: On straight sections the mobilizable friction can be used entirely for the longitudinal braking procedure. The acceleration is expressed as a fraction of $g$, the acceleration of gravity $\left(g=9.81 \mathrm{~m} \cdot \mathrm{s}^{-2}\right)$. The maximum longitudinal acceleration is:

$$
\operatorname{Acc}_{\text {Lon }}(x)=\operatorname{Acc}_{\text {Tot }}(x)=-g(\mu(x)+s(x))
$$

where $x$ is the curvilinear abscissa on the road, $\mu(x) \in[0,1]$ is the friction according to abscissa, $s(x)$ is the slope rate according to position (positive for upslope).

The actual acceleration resulting from braking is computed from the mobilized longitudinal acceleration with:

$$
A c c_{M o b}(x)=\gamma A c c_{L o n}(x)=-\gamma g(\mu(x)+s(x))
$$

where $\gamma \in[0,1]$ is a parameter linked to the driver's pressure on the brake pedal and the presence of ABS in the vehicle. $\gamma=0.9$ is used when the car has ABS and $\gamma=0.7$ otherwise [38], [39].

2) Braking in curves: While braking on straight sections allows the driver to mobilize all friction and energy in stopping, braking in curves requires mobilizing part of the friction in order to follow the path. The stopping distance is thus longer than on straight parts of the road. A model of the vehicle dynamics is used in order to account for trajectory keeping. Lateral acceleration depends on the curvature of the road and on the speed. Maximal lateral acceleration is defined as:

$$
\operatorname{Acc}_{\text {Lat }}(x)=\left|\frac{V(x)^{2}}{R(x)}(\mu(x)+\varphi(x))\right|
$$

where $R(x)$ is the radius of the curve depending on curvilinear abscissa (positive in left turns, negative in right ones) and $\varphi(x)$ is the superelevation angle (positive if the center of lane is higher than the outside). This corresponds to the part of friction mobilized in order to keep the trajectory. Given a certain amount of friction available, the total deceleration is bounded:

$$
A c c_{\text {Tot }}(x)=\sqrt{A c c_{\text {Lon }}^{2}(x)+A c c_{\text {Lat }}^{2}(x)}
$$

The mobilizable longitudinal acceleration that remains after braking is:

$$
\operatorname{Acc}_{\text {Lon }}(x)=-\sqrt{\left(g(\mu(x)-s(x))^{2}-\left(\frac{V(x)^{2}(\mu(x)+\varphi(x))}{R(x)}\right)^{2}\right.}
$$

Like on straight lines, the real deceleration produced by braking is computed by correcting the mobilized longitudinal 
acceleration with the brake pedal pressure and ABS related parameter $\gamma$ :

$A c c_{M o b}(x)=-\gamma \sqrt{\left(g(\mu(x)-s(x))^{2}-\left(\frac{V(x)^{2}(\mu(x)+\varphi(x))}{R(x)}\right)^{2}\right.}$

Notice that (8) reduces to (4) on straight sections where $R(x)=\infty$.

3) Braking Speed Profile: The mobilized longitudinal acceleration during braking at each position along straight sections and curves is known with (4) and (8) respectively. The accurate speed profile during braking $V_{B r a k}(x)$ is computed given a step $d x=1 \mathrm{~m}$ with an iterative procedure. For each position until $V(x)=0$ (i.e. the vehicle has completely stopped) the following equation is solved:

$$
\frac{V_{x+d x}^{2}-V_{x}^{2}}{2 d x}=\operatorname{Acc}_{M o b}(x)
$$

Knowing the static characteristics of the road ahead $R(x)$, $s(x), \varphi(x)$, given fixed parameters linked to vehicle and driver $t_{P R}$ and $\gamma(x)$, for a given position $x$ on the road, a braking speed profile can be computed using (9). The complete profile of speed during an emergency braking is then composed between the initial position and the total stopping distance $\left(D_{S}\right)$ as shown in Fig. 2. The total stopping distance $D_{S}$ covered from start of the emergency situation to the complete stop of the vehicle is such that:

$$
D_{S}=D_{\text {Reac }}+D_{\text {Brak }}
$$

As an example, we can foresee the influence of wet road on road safety. Wet road is a major issue for traffic safety as it means reduced pavement friction: French statistics of crashes [5] show an increase in the number of accidents under rainy conditions. Though drivers are often confronted to those conditions, they are not aware of the impact of different rainy conditions on braking distance and trajectory keeping. The speed profile is the concatenation of a constant speed part of length $D_{\text {Reac }}$ and the braking profile $V_{B r a k}(x)$ of length $D_{B r a k}$ as illustrated on Fig. 2. It illustrates different speed profiles computed for the same driver $\left(t_{P R}=1.2 s\right)$ on a car with and without $\mathrm{ABS}$ in increasing rainy conditions. The total stopping distance is almost doubled depending on the presence of $\mathrm{ABS}$ and the intensity of rain.

\section{Deducing Risk Level}

Fig. 3 shows the probability of slight, serious and fatal injury $\left(P I_{S l}, P I_{S e}\right.$ and $\left.P I_{F a}\right)$ computed for the emergency braking superimposed on the figure as a dashed black line with the curves of probability of injury $(P I)$ shown on Fig. 1. The different estimations of $P I$ are computed according to (1) with the coefficients given in Tab. I. The probability of injury for a crash ahead of the vehicle is:

$$
P I_{\text {Severity }}(x)=P I_{\text {Severity }}\left(V_{\text {Brak }}(x)\right)
$$

Fig. 3 shows how the resulting probability of injury is computed for a given profile of $V_{\text {Brak }}$. Such probability of injury curves can be computed for the three levels of severity at each

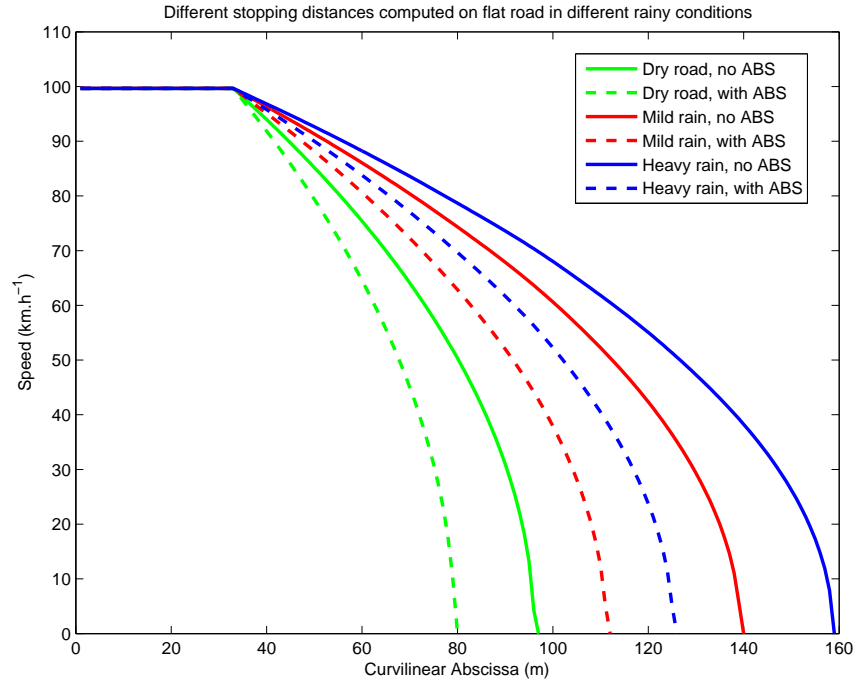

Fig. 2. Emergency braking speed profiles under various rainy conditions.

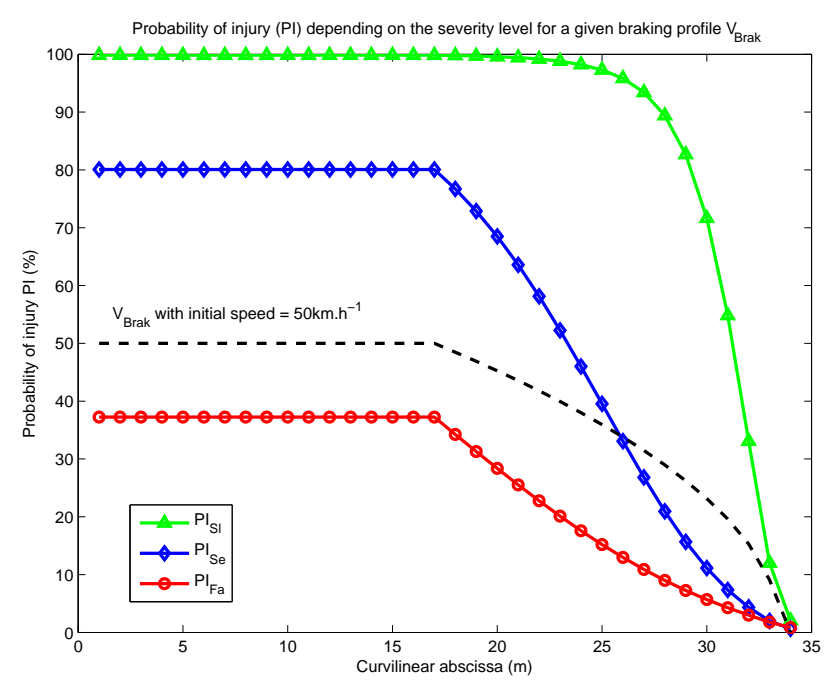

Fig. 3. Braking speed profile $V_{B r a k}$ with parameters $\left(V_{0}=50 \mathrm{~km} . \mathrm{h}^{-1}\right.$, $\left.t_{P R}=1.2 s, \mu(x)=\mu_{d r y}(x) \in[0.8,0.9], \gamma=0.9\right)$ and corresponding curves of probability of injury $P I_{S l}, P I_{S e}, P I_{F a}$.

instant on the road. If such an emergency situation occurs right in front of the vehicle, this means that the probability of incident or accident is equal to 1 . By considering the definition of highway risk given in section II-A, we can state that the risk level at a given abscissa equals $P I$.

\section{Risk Mitigation Method}

In the previous section, the highway risk has been mathematically defined. The principle of our method is then to keep the risk for the driver in adverse conditions equal to the reference risk (i.e. the risk in good weather conditions). In this aim, two speed profiles during Emergency Braking are computed. One profile is computed with good friction and good visibility conditions and the other one is computed with adverse conditions detected in real-time (such as rain that may 
reduce friction and/or visibility or such as fog that impacts mainly on visibility).

\section{A. Existing Approaches}

Existing approaches like [20], [21] focus on stopping before hitting an obstacle on the road. This strategy of handling risk has three major issues. First, in an emergency situation, the driver may run off-road in the vicinity of an obstacle and hit it with high speed. Distant obstacles are not the most dangerous ones. The second issue is that trying to avoid any contact with the obstacle can be considered as a "zero risk" strategy, while a low speed crash may not be of severe consequences. The third issue is that a too cautious strategy may end up advising a speed much lower than the legal speed, thus lowering the credibility of the advise and thus lessening the efficiency of the ISA.

The use of a "zero risk" strategy is illustrated in Fig. 5 on wet road and in Fig. 7 in fog. On wet roads, this approach would lead to lowering the initial speed so that the stopping distance $d_{S-C u r}$ is the same as on dry road $d_{S-R e f}$. In fog, this approach would lead to lowering the initial speed so that the stopping distance $d_{S-C u r}$ equals the visibility distance $d_{V i s}$.

\section{B. Novel Approach}

Our novel approach consists in lowering initial speed in adverse conditions (reduced friction or reduced visibility) in such a way that the total probability of injury in adverse conditions equals the total probability of injury in reference conditions, all else being equal:

$$
\int_{0}^{d_{S-R e f}} P I\left(V_{\text {Brak-Ref }}\right) d x=\int_{0}^{d_{S-C u r}} P I\left(V_{\text {Brak-Adv }}\right) d x
$$

In the following sections we show the result of this "Equivalent Total Risk" (ETR) approach at one point of the road in rain and in fog for a given severity level.

\section{Risk Mitigation in Rain - One severity level}

The ETR strategy is illustrated in rainy weather in Fig. 4 and Fig. 5 at one point of the road. A dichotomic procedure is applied to find an initial speed in adverse conditions such that the total probability of fatal injury during braking is equivalent to the total reference probability of fatal injury as proposed in 12 , all else being equal.

Fig. 4 presents the fatal $P I$ curve in reference conditions for a driver at reference speed $V_{R e f}=90 \mathrm{~km} . \mathrm{h}^{-1}$, on a dry road with $\mu_{R e f} \in[0.83,0.88]$ on this section of the road (plain blue line). At first, the fatal $P I$ (dotted red line) for the current conditions is estimated with $V_{C u r}=90 \mathrm{~km} \cdot \mathrm{h}^{-1}$ on a wet road with $1 \mathrm{~mm}$ water height $\left(\mu_{C u r} \in[0.47,0.51]\right)$. If the total current $P I_{F a-C u r}$ is superior to the reference $P I_{F a-R e f}$, an advisory speed $V_{A d v}=81 \mathrm{~km} \cdot \mathrm{h}^{-1}$ is computed such that total $P I_{F a-A d v}$ with advisory speed (dot-dashed green line) equals total reference $P I_{F a-R e f}$. Notice that with our advisory speed, the stopping distance is slightly longer than in the reference conditions ( $93 \mathrm{~m}$ versus $79 \mathrm{~m}$ ).

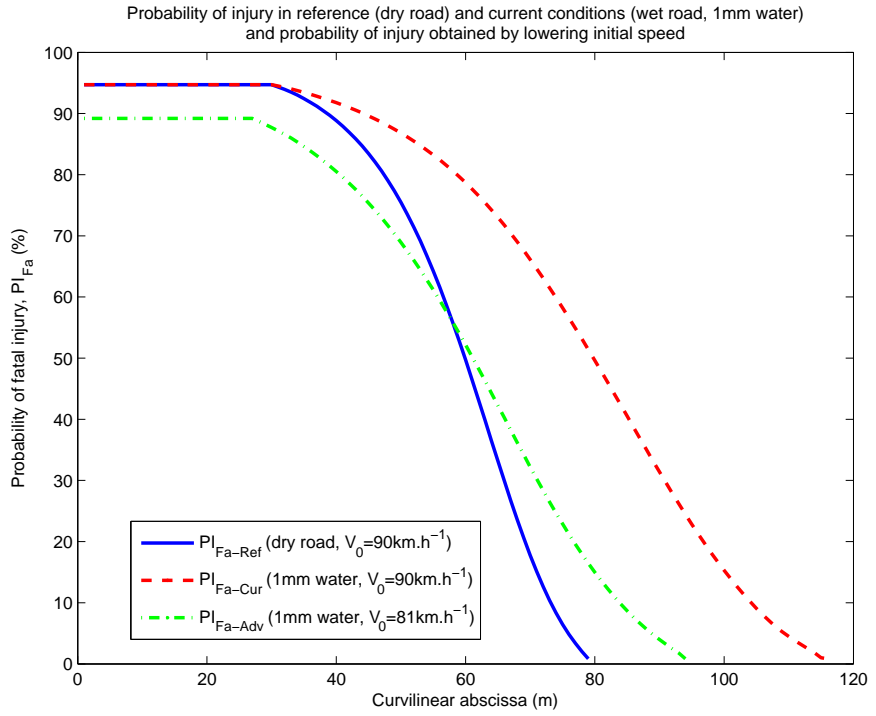

Fig. 4. Probability of fatal injury $P I_{F a}$ for emergency braking on wet pavement at $90 \mathrm{~km} \cdot \mathrm{h}^{-1}$.

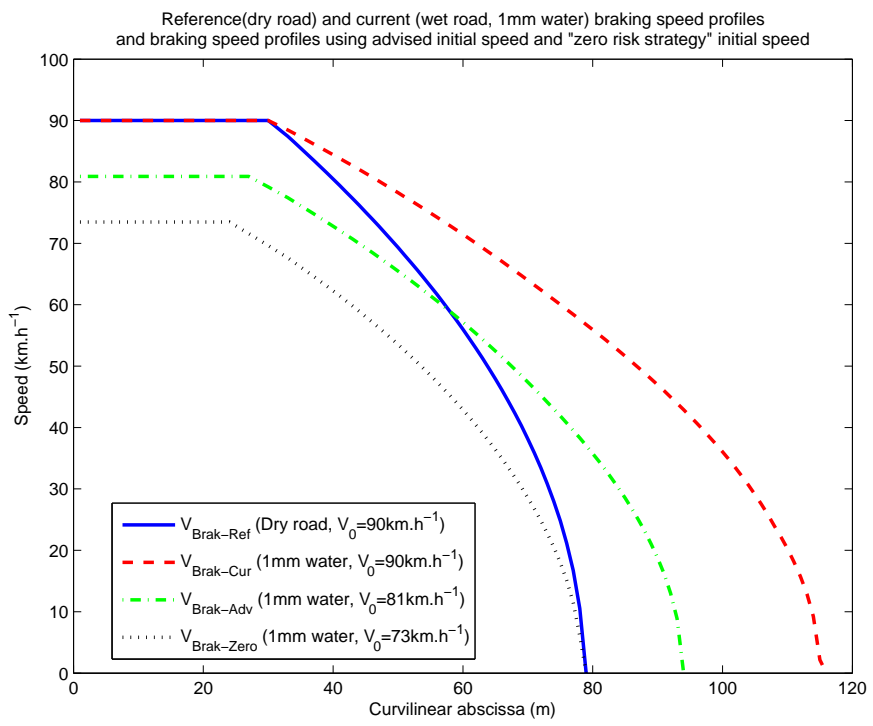

Fig. 5. Emergency braking speed profiles in rain at one point on the road.

Fig. 5 presents the different emergency braking speed profiles that would occur in the different conditions and using the different advisory strategies. The braking speed profile using the "zero risk" strategy such as proposed in [20], [21] (dot black line) is also presented for comparison. Using this method leads to an advisory speed such that the stopping distance equals the reference stopping distance $(79 \mathrm{~m})$. As expected, such a strategy would lead to the lowest advisory speed $\left(V_{\text {Zero }}=73 \mathrm{~km} \cdot \mathrm{h}^{-1}\right)$.

At any given point along the road, the risk in the current situation and the risk in the reference situation are compared. From this comparison a lower speed can be proposed which brings the current risk down to the same level as the reference risk. It is interesting to note that the advised speed is actually that recommended in the driving manuals for rainy weather 


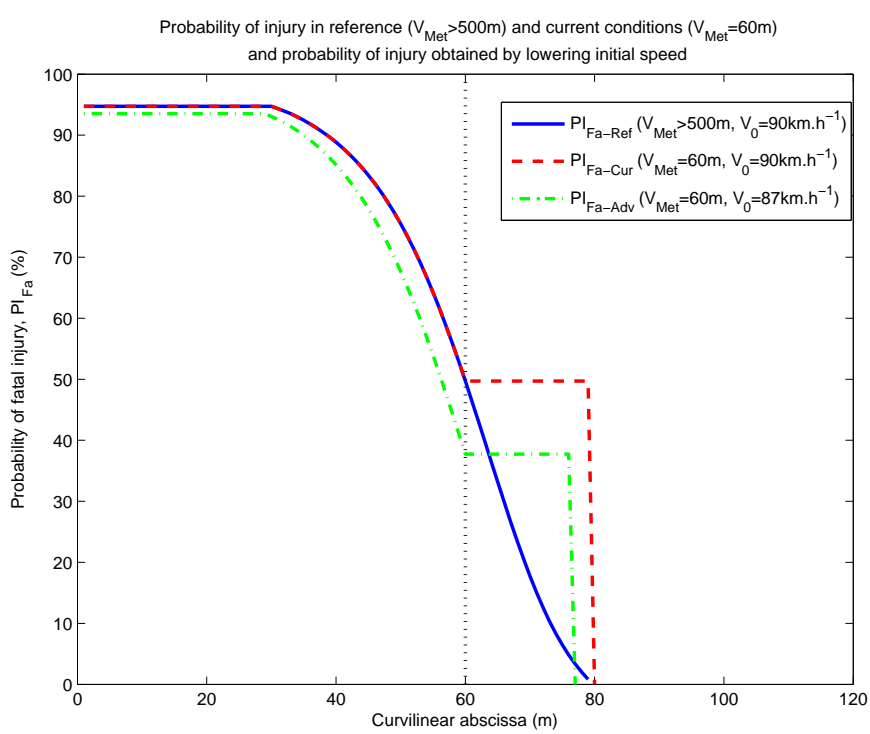

Fig. 6. Braking speed profile and probability of fatal injury in fog with $60 \mathrm{~m}$ of visibility distance.

on rural highways $\left(90 \mathrm{~km} \cdot \mathrm{h}^{-1} \rightarrow 80 \mathrm{~km} \cdot \mathrm{h}^{-1}\right.$ ). Our strategy can be applied for each level of severity, giving three different advised speeds with which the total risk in adverse conditions of friction equals the total risk in reference conditions for that level of severity.

\section{Risk Mitigation in Fog - One severity level}

Fog essentially impairs highway visibility. French statistics of crashes [5] show that it increases the average severity of crashes, meaning drivers collide with obstacles at higher speeds. Potential severity calculations are adapted by considering that the probability of injury is constant beyond the visibility distance as shown in Fig. 6. The probability of injury depending on severity is set constant from the visibility distance up to the total stopping distance. This has no impact if the braking speed profile produces a total stopping distance lower than the visibility distance. Our ETR strategy is applied at this point of the road and a dichotomic procedure is then applied to find an initial speed in adverse conditions such that the total probability of fatal injury during braking is equivalent to the total reference probability of fatal injury, all else being equal.

Fig. 6 presents the fatal $P I$ curve in the reference conditions (plain blue line) for a driver at reference speed $V_{R e f}=$ $90 \mathrm{~km} . \mathrm{h}^{-1}$, on a dry road with $\mu_{R e f} \in[0.83,0.88]$. In this example, considering that the fog only impacts on visibility, the current braking speed profile is identical to the one in the reference conditions $V_{B r a k-C u r}=V_{B r a k-R e f}$. Nevertheless, the current fatal $P I$ computed for that braking profile is modified such that $P I_{F a}\left(x>V_{M e t}\right)=P I_{F a}\left(V_{M e t}\right)$ (dashed red line). The total current $P I_{F a-C u r}$ being superior to the reference $P I_{F a-R e f}$, an advisory speed $V_{A d v}=87 \mathrm{~km} . \mathrm{h}^{-1}$ is computed such that the total $P I_{F a-A d v}$ with advisory speed (dot-dashed green line) equals the total reference $P I_{F a-R e f}$.

Fig. 7 presents the different emergency braking speed profiles that would occur with the reference and advised

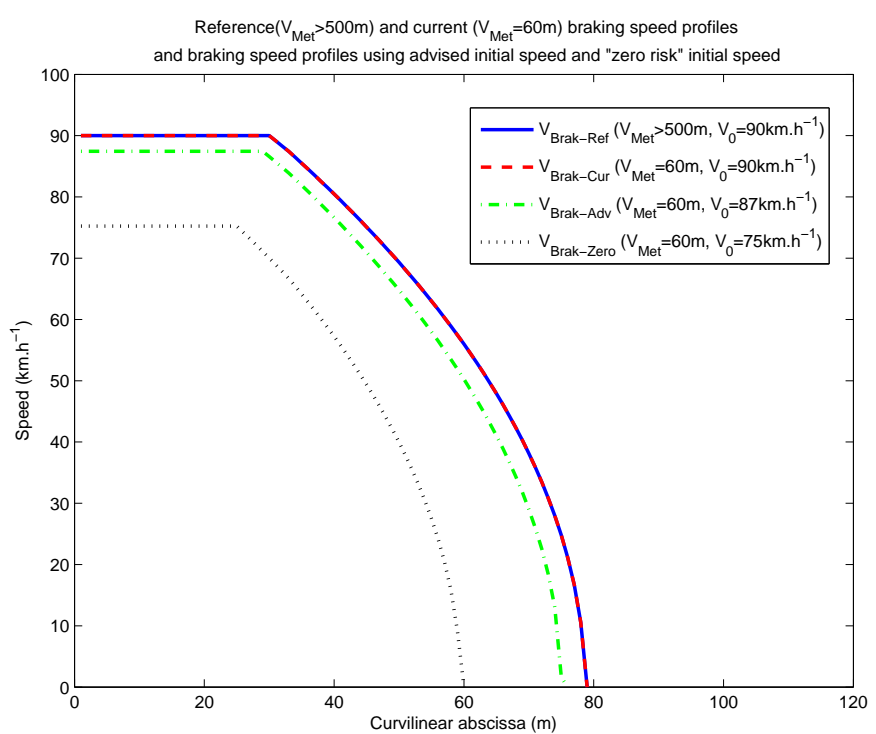

Fig. 7. Emergency braking speed profiles in fog at one point on the road.

speeds computed using the different strategies. The profile resulting from using the "zero risk" strategy (dot black line) is also presented for comparison. Using this method leads to an advisory speed such that the stopping distance equals the meteorological visibility distance $\left(V_{M e t}=60 \mathrm{~m}\right)$. As expected, such a strategy would lead to the lowest advisory speed $\left(V_{\text {Zero }}=75 \mathrm{~km} \cdot \mathrm{h}^{-1}\right)$.

At any given point along the road, the risk in the current situation and the risk in the reference situation are compared. From this comparison a lower speed can be proposed in order to bring the current risk down to the same level as the reference risk. Our strategy can be applied for each level of severity, giving three different advised speeds with which the total risk in adverse conditions of visibility equals the total risk in the reference conditions for that level of severity, all else being equal.

Using the method exposed in the previous sections for rainy or foggy situations leads to different advised speeds depending if one focuses on ETR for slight, severe or fatal injuries. We illustrated our strategy with the fatal injury criterion but the method is identical with other criterions.

We next show how we compose different speed recommendations depending on the severity of injury criterion considered.

\section{Risk Mitigation along a RoAd Section}

In the previous section, a risk mitigation strategy, called ETR, has been presented and applied at one point of a road in case of reduced friction or visibility. In this section, the ETR strategy is applied on an actual road section with simulated weather conditions. A road itinerary of $40 \mathrm{~km}$ was selected in the network of the Conseil Général (CG 22) of Côtes d'Armor (RD 786) in West of France. On this section, infrastructure parameters were collected and a $V_{85}$ speed profile was measured in ideal conditions to be used as reference speed profile. 


\section{A. Estimation of a Reference Speed}

The $V_{85}$ speed is the $85^{\text {th }}$ percentile of a speed distribution, it is usually measured in ideal meteorological conditions. This definition is widely used in roadway engineering, either for design or safety purposes although it depends on the way it is measured. It depends on the nature of vehicles taken into account (light vehicles, trucks etc.) and also if the vehicle is in traffic or in free-flow (3 to 10 seconds from preceding vehicle). Its estimation could be erroneous if the moment and the integration time of the measurements are not carefully chosen. This speed is praticed and practicable in the same conditions as the measuring conditions (by light vehicles in free flow in our example).

Unlike usual measures of $V_{85}$ collected at one spot, a continuous profile of $V_{85}$ along a path is used. This profile of reference speed can be computed with the methodology presented in [40]. In order to get this profile, several realdriving sessions are needed during day and night. Profiles where the measuring car is constrained by traffic need to be discarded (night sessions are easier in this respect). The test drivers are asked to follow the path with either normal or hurried speed. Fig. 8 presents two extreme profiles observed (the slowest one and the fastest one), the posted speed limit and the estimated $V_{85}$.

On secondary roads, for a vehicle in free flow conditions with ideal meteorological conditions, the $V_{85}$ is a more realistic and acceptable speed to be advised than the regulatory speed. For legal purposes, it may be necessary for the reference speed to comply with posted speed limits. The reference speed to be used can be defined as the estimated $V_{85}$ as long as it is lower than the posted speed limit.

\section{B. Collection of Infrastructure Parameters}

Geometric characteristics of the roads are of major importance concerning the dynamic behavior of the vehicles. Many "run off-road" crashes are the consequences of a misunder-

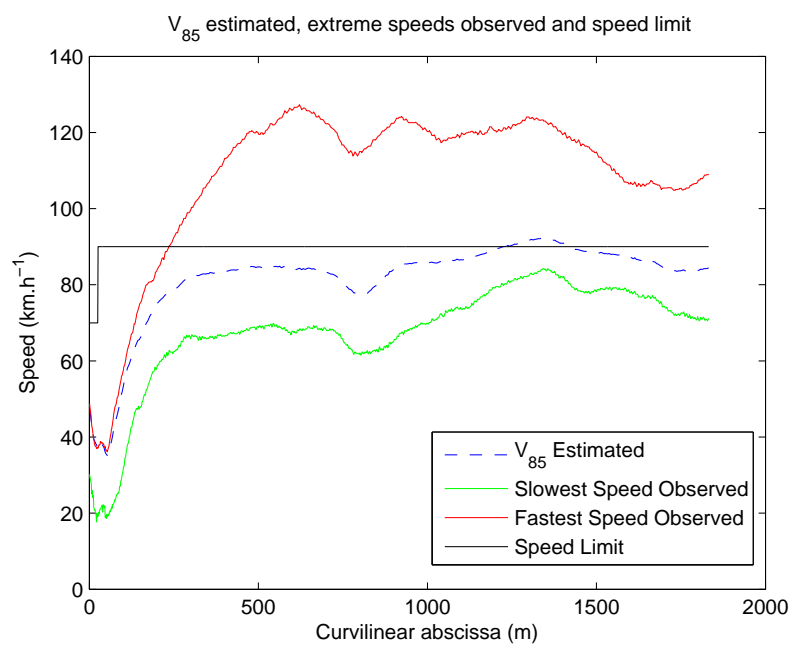

Fig. 8. Estimated $V_{85}$ speed profile, extreme speeds observed and speed limit on a portion of the RD786 of CG 22 (France). standing of the complex interaction between those characteristics and vehicle speed [4]. For example, most drivers don't know the impact of slope on the length of braking or the limits of controllability of their vehicle depending on their speed with respet to the road curvature in curves. A mapping vehicle can be driven along a road to gather information such as curvature, slope angle and superelevation. These characteristics do not evolve in time. Once acquired this information is available for the whole road. It could be embedded into cars through the use of maps. It could also be transmitted by the infrastructure using relevant V2I protocols. An electronic horizon of $300 \mathrm{~m}$ is sufficient in order to compute emergency braking profiles according to the methodology detailed in section III.

All the characteristics are not alike. Friction, which is a major characteristic of the road, is mainly linked to road roughness, tyre nature (slick or engraved) and water height. Our measuring vehicle is able to estimate friction for a standard tyre under $1 \mathrm{~mm}$ of water. This characteristic is assumed to be semi-static. The profile of friction can be transmitted to the car along with other characteristics of the road. Water height will be measured by roadside units thanks to dedicated devices and spatially refined in real-time with cameras using algorithms such as [25], [26], [41]. Then, using a model of friction estimation depending on water height, real friction can be estimated at any time in the vicinity of the car [42].

\section{First Results of Risk Mitigation along a Road Section}

Fig. 9 shows the result of the methodology that leads to an advisory speed with our ETR strategy. Using this methodology allows us to propose, at a given position on the road, three different advisory speeds depending on the potential severity taken into account. Accounting for "slight injuries" leads to a more cautious speed than accounting for "fatal injuries". The advisory speeds computed with our method will be compared

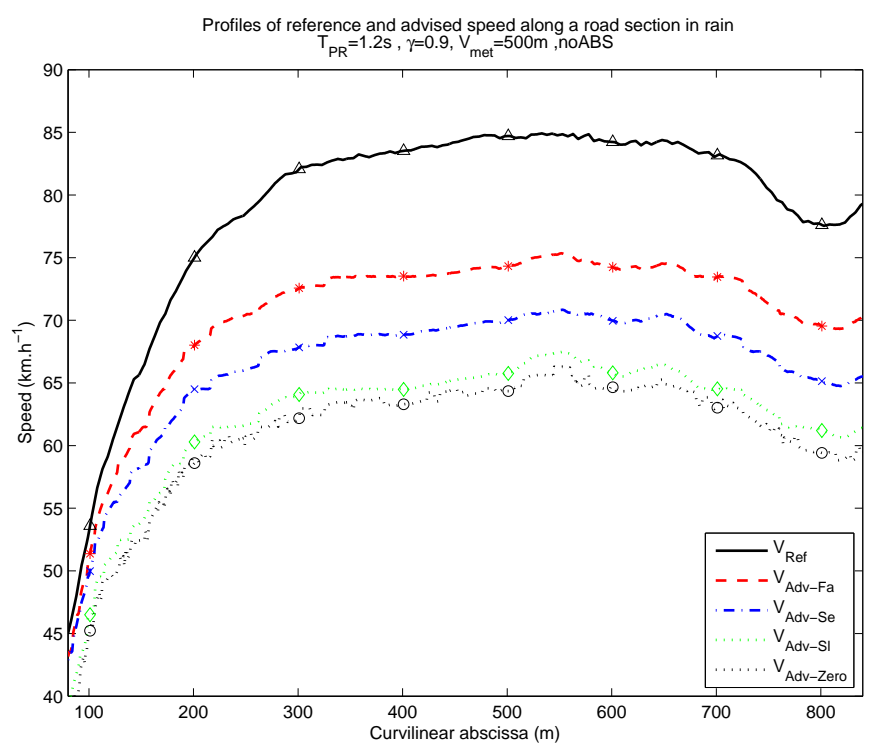

Fig. 9. Profiles of advised speeds computed with different safety criterions on wet road. 
to the reference speed on dry road (i.e. the $V_{85}$ speed in good weather, or $V_{R e f}$ ) and to the advisory speed computed with an existing method based on the "zero risk" strategy.

It is possible to compute different braking profiles under rainy conditions, it is also possible to compose these braking profiles with the curves of probability of injury in order to propose an advisory speed along the road as shown in Fig. 9.

Notice that the advisory speed taking into account slight severity injuries is very close to the advisory speed proposed with the "zero risk" strategy proposed by [20], [21]. This was expected because slight injury curves are very sharp for low speeds and saturated over $10 \mathrm{~m} . \mathrm{s}^{-1}$ (See Fig. 1). This means that only the end of the braking speed profile is used to compensate for the ETR strategy. The "zero risk" strategy can be assimilated to an ETR strategy with a severity curve equal to $100 \%$ from $\Delta V=1 \mathrm{~m} . \mathrm{s}^{-1}$ (100\% probability of severity whatever the speed of the crash, meaning the crash should be absolutely avoided).

\section{Risk Mitigation for different severity levels}

In Fig. 3, the braking speed profile goes from a medium speed $\left(50 \mathrm{~km} \cdot \mathrm{h}^{-1}\right)$ down to stop and the different probabilities of injury between $37 \%$ and $100 \%$ at the beginning of the emergency braking down to $0 \%$ at the end. This is the direct consequence of the application of the curves shown in Fig. 1. This means that applying this composition on an emergency braking at high speed would lead to a $P I_{S l}$ near $100 \%$ along most of the braking distance. Conversely, using such composition on a low speed emergency braking ranging from $30 \mathrm{~km} . \mathrm{h}^{-1}$ to stop would lead to a $P I_{F a}$ near $0 \%$ along most of the braking distance.

This reflects the fact that at high speeds, whatever the distance from the obstacle, the driver is highly susceptible to have at least a slight injury and that at low speeds, it is highly improbable that he might suffer fatal injuries. Seeing

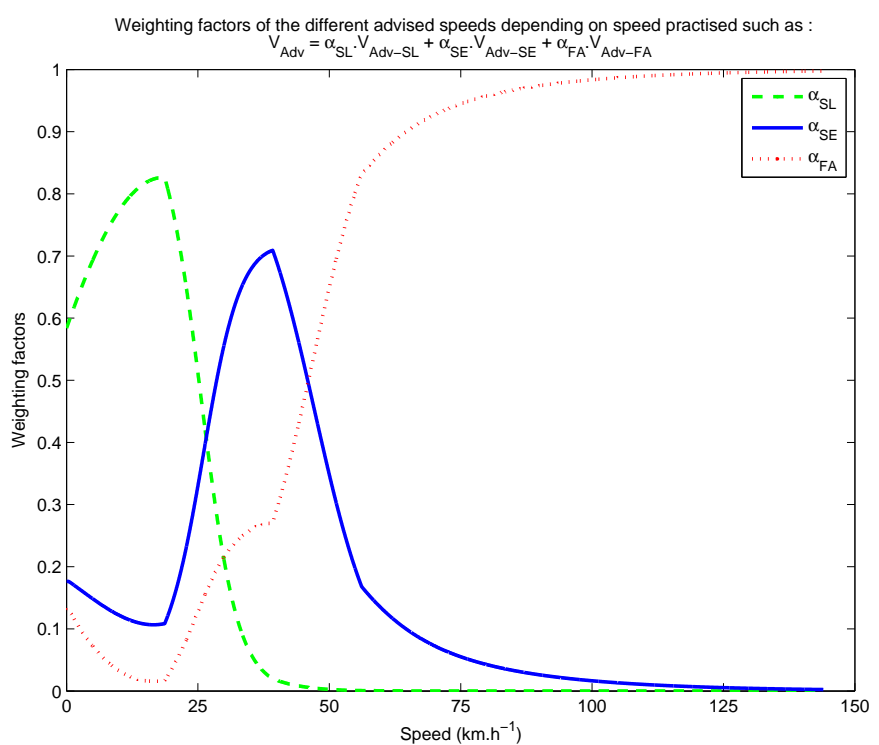

Fig. 10. Weighting of advised speeds linked to different severity levels. this evidence, we consider that it is not of major importance to modulate the initial speed for fatal injuries when there is almost no risk of fatal injury and for slight injuries when they are almost unavoidable. We automatically compose the advised initial speeds corresponding to different severity of injury criteria by accounting for the most relevant criterion is emphasized. The weighting of the advised speed is computed according to (13):

$$
\begin{gathered}
\alpha_{\text {Severity }}=\frac{\min \left(\overline{P I_{\text {Severity }}}, 100-\overline{P I_{\text {Severity }}}\right)}{\left(\min \left(\overline{P I_{S l}}, 100-\overline{P I_{S l}}\right)+\right.} \\
\min \left(\overline{P I_{S e}}, 100-\overline{P I_{S e}}\right)+ \\
\left.\min \left(\overline{P I_{F a}}, 100-\overline{P I_{F a}}\right)\right)
\end{gathered}
$$

where Severity stands for one of the severity criterions considered (slight, serious or fatal), and $\overline{P I_{\text {Severity }}}$ is the mean value of $P I$ over the emergency braking distance.

Using (13), we compute at each point of the road three weighting factors, $\alpha_{S l}, \alpha_{S e}$ and $\alpha_{F a}$ whose sum equals 1 and we advise a current speed recommendation according to (14):

$V_{A d v-f i n a l}=\alpha_{S l} . V_{A d v-S l}+\alpha_{S e} . V_{A d v-S e}+\alpha_{F a} . V_{A d v-F a}$

Fig. 10 shows the value of the weighting factors $\alpha$ used to weight the different advised speeds corresponding to different severity criteria depending on the current speed.

We showed how the advised speed can be computed at each point of the road depending on different adverse conditions and accounting for different levels of severity of injury. We showed how we compose the different advised speeds computed in order to propose a unique advised speed to the driver. In the next section the whole profiles of the advised speed along the road are presented depending on different adverse conditions.

\section{E. Experimental Results}

1) Reduced friction on wet road: We show in Fig. 11 the profile of our advised speed composed from the three advised speeds shown in Fig. 9. At low speed, the profile tends to be closer to the speed advised to prevent slight injury whereas at high speed, it is closer to the speed advised to prevent fatal injury.

2) Reduced visibility in fog: As shown in Fig. 11 for rainy situations, we computed three different advised speeds in fog using different safety criteria. The advisory speeds along the whole road are computed in the presence of fog limiting visual range to $50 \mathrm{~m}$ as shown in Fig. 12.

We computed a weighted advised speed according to the methodology presented in Sec. IV-D and as we did in Fig. 11 for a rainy situation. The final advised speed in fog is shown in Fig. 13

Notice that since the visual range is limited to a fixed distance, the speed proposed using the "zero risk" strategy is almost constant. The small variations are only due to road properties used in our model of the dynamic of the car $(p(x)$, $\mu(x)$ and $\phi(x))$. These small variations allow for the driver to always be able to stop within $50 \mathrm{~m}$. Before an abscissa of $150 \mathrm{~m}$, none of the strategies ("total risk" or "zero risk") 


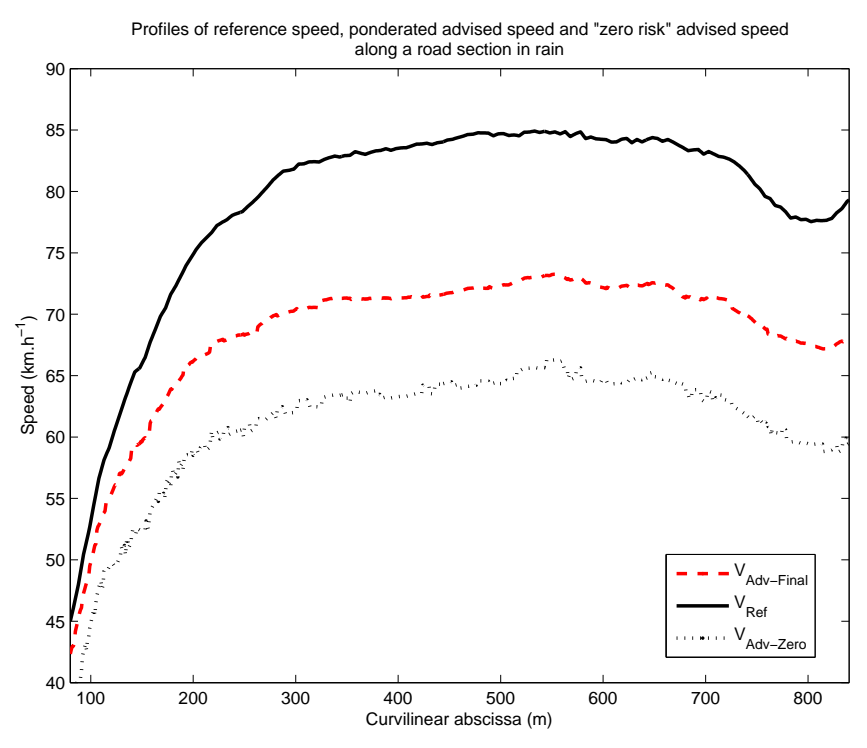

Fig. 11. Speed profiles under rainy conditions.

have any impact on the initial speed since the initial speed is sufficiently low to allow for the driver to stop in less than 50 m.

We showed how we handle different adverse conditions independently but the ETR strategy presented in this article can of course be applied to rainy and foggy conditions simultaneously, rain having a strong impact on the braking distance while fog impacts the probability of accident beyond the visibility distance.

In the next section we present the tests we conducted on our test site in order to assess the feasibility of our method.

\section{System ImPlementation AND Demonstration}

In the previous section, the ETR strategy has been applied offline with data from an actual highway. In this section, a real-

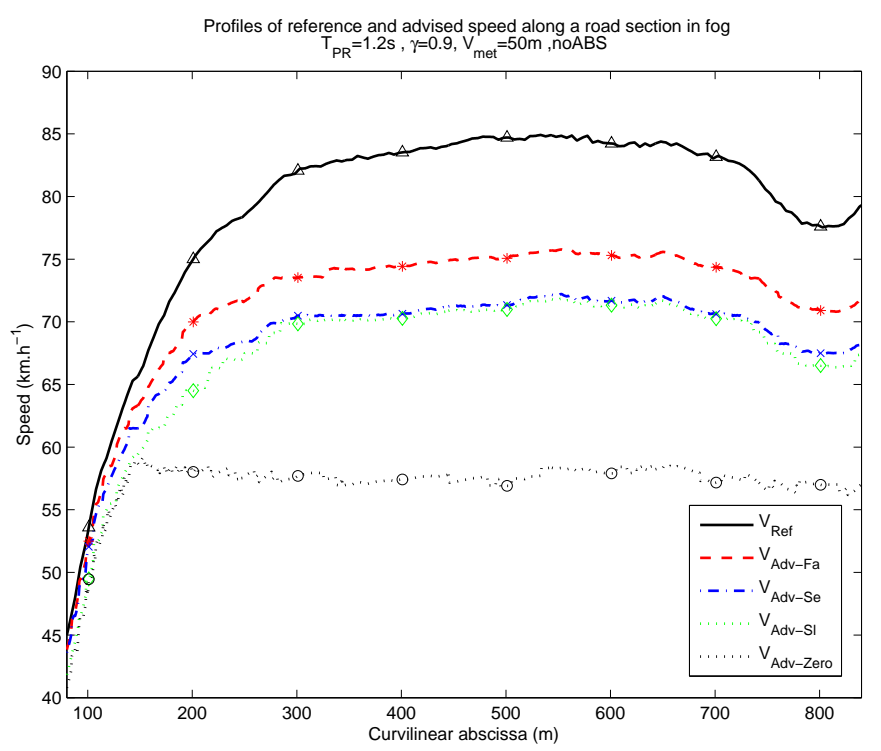

Fig. 12. Different speed profiles in fog with $V_{M e t}=50 \mathrm{~m}$.

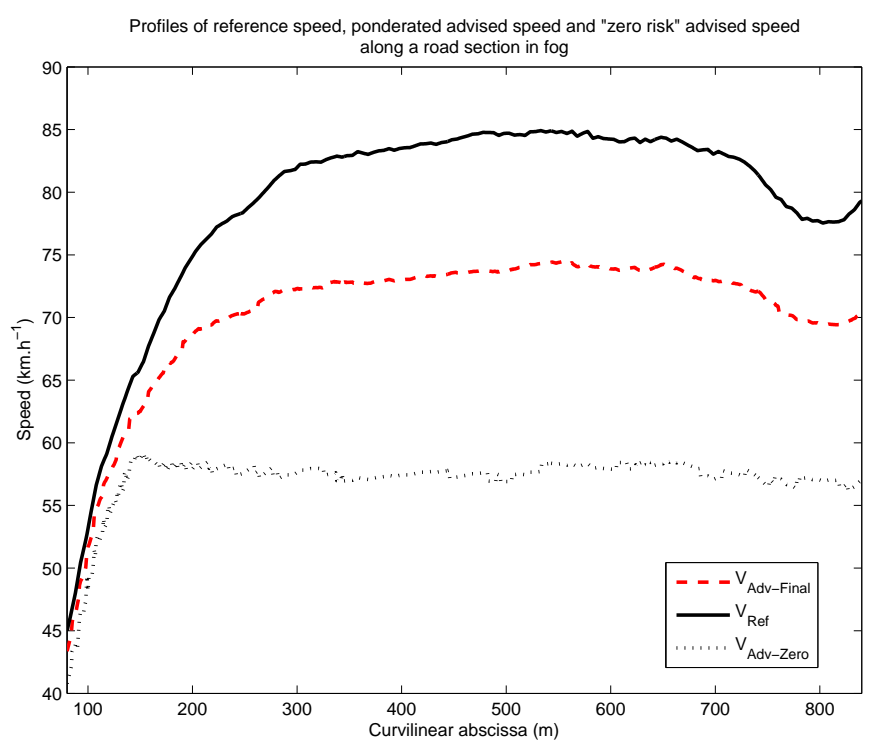

Fig. 13. Reference, "zero risk" and ETR speed profiles in fog with $V_{M e t}=$ $50 \mathrm{~m}$.

time implementation of this ETR strategy is proposed in the framework of the French DIVAS project initiated in 2007 [43]. First, the system is described as well as the experimental test site. Second, the equipment of the prototype vehicles is presented. Third, the outputs of the system in four different scenarios are given. Finally, the acceptability of the system is tackled.

\section{A. The Divas System}

The global scheme of this Vehicle Infrastructure Integration (VII) project is presented in Fig. 14. The present article presents the in-vehicle comparison that is done in order to adapt speed to road characteristics (curvature, slope, superelevation), vehicle characteristics (parameters such as the presence of ABS or possibly tire quality) and environmental parameters that can only be estimated in real-time (such as wet road or limited visibility conditions triggered by rain, fog or snow). Road characteristics fall within the competence of road operators, as they are relatively permanent. They are transmitted to the car by servers next to the road. In this section, the implementation of the system is presented as well as the tests carried out on the test site of IFSTTAR in Nantes (France) to prove the technical feasibility of the system.

\section{B. Test Site Description}

To test and prove the feasibility of the DIVAS system, different tests were carried out. The most complex one took place on the test track of IFSTTAR in Nantes. This test track presents curves with different radii and road surfaces with different friction coefficients depending on the section. The test track was equipped with water sprinklers in order to simulate rain on one section, a fog machine in order to simulate fog and maskings on the road side in some curves in order to simulate a local reduction of the geometric visibility as illustrated on Fig. 15. The track was also equipped with five 
wireless access points which are autonomous from the energy point of view thanks to solar cells. The weather station which equipped the test site was connected to the ad-hoc network. A roadside unit (RSU) was installed to monitor the test track. The characteristics of the test track were acquired by the CETE de Lyon using a dedicated mapping vehicle. The static data were map-matched and introduced into the RSU and the On-Board Unit (OBU). As there was no traffic on the test site, no $V_{85}$ profile was available and the reference speed profile issued from the SAVV model [44] was used instead. Fig. 16-a shows the relevance and potential utility of a model of practiced speed such as SAVV when a reference profile cannot be estimated using the methodology exposed in Sec. IV-A.

\section{Equipment of the Vehicles}

To demonstrate the interest of V2I communications, two types of OBU were implemented. The first one is merely a Netbook PC with a simple GPS unit. This simple OBU does not have access to the sensors of the vehicles and is representative of a personal navigation device. The second one is an expert OBU. It is integrated with the different sensors of the vehicle: camera, odometers, etc. This type of OBU is able to collect exteroceptive data such as rain or fog presence. It is representative of an integrated navigation system. Both types of OBUs were developed by teams from two different research institutes based on open standards. They were able to exchange data correctly with the same RSU. Five different vehicles were equipped, including a coach for public transport. The four cars could thus continuously and simultaneously transport observers to demonstrate the concept while the coach could demonstrate for groups of people.

\section{Test Scenarios}

To show the effectiveness of the DIVAS system, four experimental scenarios were designed which made use of the different subsystems and involved the five prototype vehicles simultaneously:

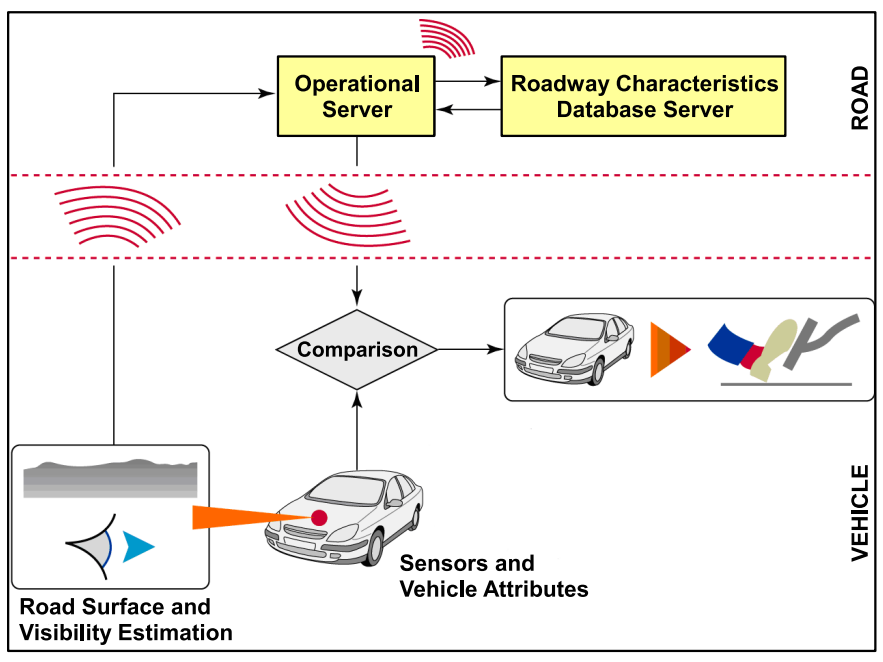

Fig. 14. Layout of Vehicle Infrastructure Integration in DIVAS Project
- The first scenario consisted in providing the driver with a speed recommendation in case of good weather and a dry road. This scenario made use of the RSU which communicates the reference speed to the vehicle. Recall that this speed depends on the road geometry, previously collected by a mapping vehicle.

- The second scenario consisted in reducing the sight distance of the driver using masking objects at the entrance of a curve. The sight distance can be computed based on 3D data collected by a dedicated mapping vehicles, following for instance the methodology proposed by [45].

- The third scenario consisted in reducing the meteorological visibility distance using a fog machine. The presence of fog was detected and the meteorological visibility distance was estimated by the expert OBU by means of the camera along with the image processing technique proposed by [22]. This information was then transmitted to the RSU which transmitted the information to simple OBUs.

- The fourth scenario consisted in reducing the skid resistance of the pavement by simulating rain with roadside sprinklers. The presence of rain was detected by an expert OBU. The information was transmitted to the RSU which estimated the water layer thickness in order to estimate the skid resistance. The result was transmitted to all OBUs which computed the final speed recommendation.

As one can see, these four scenarios make use of the different subsystems of Divas. They are illustrated in Fig. 15. On the left side of the figure, the scenario is recalled and on the right side of the figure, a picture of the running demonstration is shown. Finally, in Fig. 16 the speed recommendations issued by the DIVAS system in the different scenarios are presented. Fig. 16-b shows the local speed modulation proposed in a curve with sight distance reduced down to $50 \mathrm{~m}$. The starting

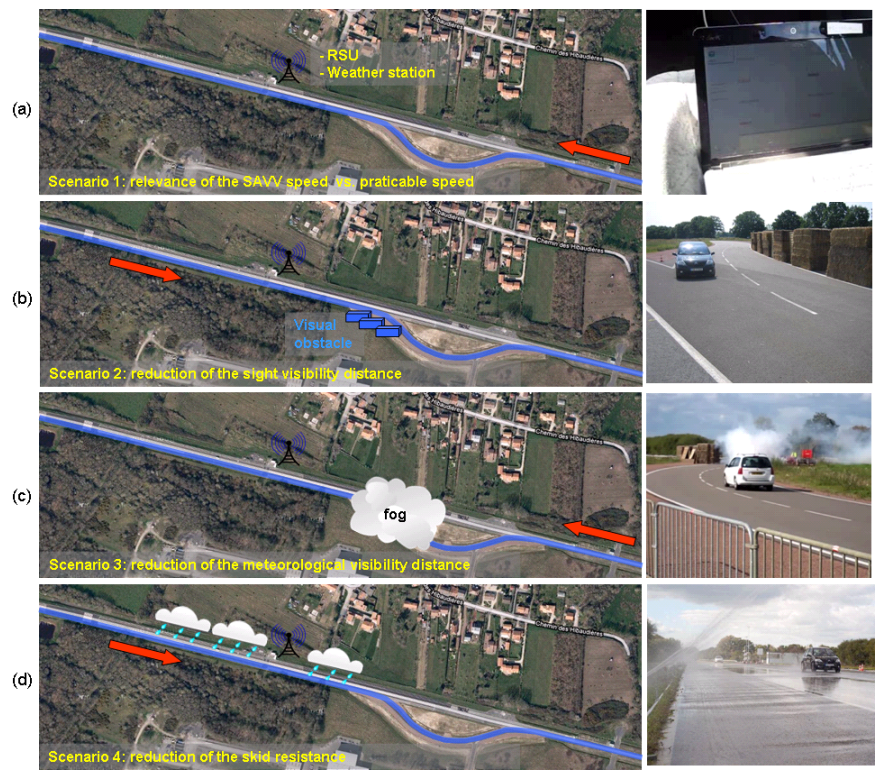

Fig. 15. Illustration of the different test scenarios: (a) relevance of the SAVV speed vs. the practicable speed; (b) reduction of the sight distance; (c) reduction of the meteorological visibility distance; (d) reduction of the skid resistance. 

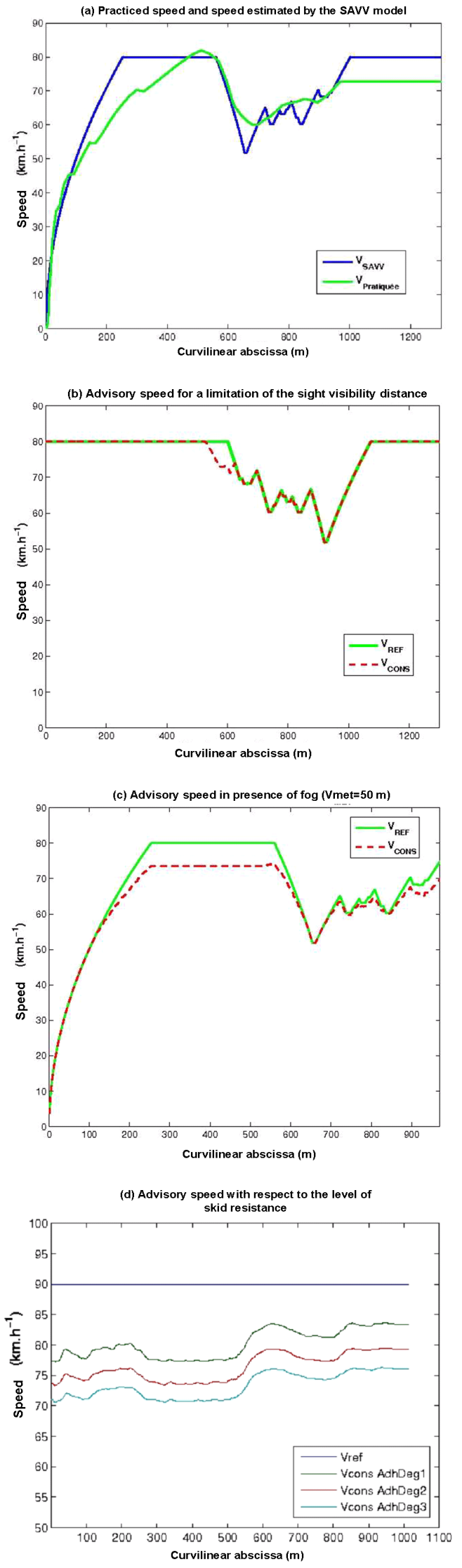

Fig. 16. Speed recommendation issued by the DIVAS system on the different test scenarios: (a) speed profile in curve; (b) speed profile in case of reduced sight distance; (c) speed profile in case of reduced meteorological visibility; (d) speed profile in case of a wet road (with different levels of skid resistance). speed on the figure is not null since in this scenario, cars are already at the speed limit before entering the straight section. Fig. 16-c shows the speed modulation along the section when fog reduces visual range. The density of the fog simulated was not constant on the whole track but the proposed speed is computed using a constant meteorological visibility distance $V_{M e t}=50 \mathrm{~m}$. Fig. 16-d shows that on a straight line, where a driver usually keeps its speed constant, the system is able to propose a speed that is modulated all along the driveway by accounting for the impact of the water on the different road surfaces. The rain simulated on the track using sprinklers was quite light but the proposed speed is computed using the measured friction of the track under $3 \mathrm{~mm}$ of water height with the measuring vehicle described in Sec. IV-B

In these scenarios, the accurate validation of the issued data was not of primary importance nor were the HMI issues. The goal was to demonstrate that every information introduced in the Divas system impacted the speed recommendation and thus in turn impacted the driving safety.

\section{E. Acceptability}

A complementary study has been carried out to analyze how the recommandations of the proposed device could be used and accepted by drivers [46]. "Acceptability" was understood as " the conditions in which the system is good enough to satisfy the needs and requirements of actual and potential users" [47]. Relying on the UTAUT model - Unified Theory of Acceptation and Use of Technology - [48], it has been shown that the will to use the device is correlated with the expected performances, the expected exertion, norms and driving styles.

\section{CONCLUSiOn AND Future Works}

This paper presents a new approach to compute an advisory speed to be used in an ISA or an ADAS, by supervising the speed limit. By using measured characteristics of the road, accurate speed profiles during emergency braking are computed. The system proposed is able to deal with multiple adverse conditions that impair friction and visibility such as rain, fog or both simultaneously. Vehicle and driver related parameters such as the presence of ABS, the pressure on the brake pedal or the perception-reaction time can be used in our model of the vehicle dynamic. The presented method is open and can be extended to specific situations such as nighttime driving. It is also adaptable to the country, network, population of drivers and vehicles, by using the relevant statistics. Finally, it is improvable by adding or modifiying models of the car dynamics, of the driver behavior or the interaction between environment, infrastructure, car and driver.

The novelty of this approach also comes from the use of a reference speed considered safe in ideal driving conditions. This speed is modulated in adverse conditions using potential accident severity criteria. Our advisory speed lies between the reference speed and the speed computed using previous works with a very cautious strategy based on the stopping distance.

In the end three different advisory speeds are computed, which could be qualified as very cautious, cautious and normal. When a speed is used in an ISA, it should be kept in mind 
that big differences from legal speed or current speed may have less impact and that the driver could be tempted to ignore the advise: Over-cautious strategies can lead to less efficiency of an ISA as shown in [49]. A much lower speed than the legal one may also result in large speed differentials among the various drivers on the road and could lead to increased risk. As our method relies on a realistic and practiced speed, not a model driven approach, the proposed modulation of speed is less susceptible of leading to high differences of speed on the road. This point is essential and should be considered along with the adaptability of the proposed method to many factors related to the environmental conditions, the infrastructure, the vehicle and the driver.

In the future, the method will further be extended using statistics for other types of crashes such as "hit rigid fixed obstacles", "hit stopped cars" and "hit facing driving cars" with curves of probability of injury corresponding to these scenarios. We have to choose a strategy for setting parameters such as perception-reaction time or pressure on the brake pedal. At the present time, these parameters are fixed considering that $t_{P R}=1.2 \mathrm{~s}$ corresponds to a good driver, or more carefully $t_{P R}=2 \mathrm{~s}$ as it is the $95^{t h}$ percentile of perceptionreaction time of drivers. This value of $t_{P R}$ used in this example is driven from the reference value chosen for road design in France. It should be noted that this parameter is dependant on many factors such as driver related parameters, environemental conditions and speed for instance and that our method allows for modification of this value provided one has the knowledge on the value of this parameter in specific conditions. Using accurate information on a specific driver could lead to advised speeds that suit the driver even better [50]. In this aim, relevant warnings are likely to improve the user acceptance [51]. If the user acceptance is high enough, the safe speed profiles could be ultimately used in Automotive Cruise Control systems in adverse weather conditions [52].

\section{ACKNOWLEDGMENT}

This work was supported by the French ANR DIVAS Project. We would like to thank CETE Normandie-Centre for providing us with speed profiles along the road and CETE Lyon for providing us with measured characteristics of the roads. The authors would like to thank Eric Dumont for having helped us in the revision of the manuscript and Philippe Lepert for his excellent management of the DIVAS project.

\section{REFERENCES}

[1] WHO, "The global burden of disease : 2004 update," tech. rep., World Health Organization, Geneva, Switzerland, 2008

[2] A. Amditis, M. Da Lio, and R. Goudy, "Guest editorial special section on ITS and road safety," IEEE Transactions on Intelligent transportation Systems, vol.11, no.3, p.524, 2010.

[3] NHTSA, "Traffic safety facts 2008," tech. rep., National Highway Traffic Safety Administration, 2008.

[4] S. McLaughlin, J. Hankey, S. Klauer, and T. Dingus, "Contributing factors to run-off-road crashes and near-crashes," Report NPO-113, NHTSA, USA, 2009.

[5] ONISR, "The major data on accidentology," tech. rep., National road safety observatory, France, 2007

[6] T.R. Centre, "Speed management," tech. rep., OECD/European Conference of Ministers of Transport, 2006.
[7] G. Nilsson, "The effect of speed limits on traffic accidents in sweden," Proc. Int. Symp. on the Effects of Speed Limits on Traffic Accidents and Transport Energy Use, Organization for Economic Cooperation and Development, 1981.

[8] R. Elvik, "Speed and road accidents: an evaluation of the power model," tech. rep., Institute of Transports Economics, 2004.

[9] D. Finch, P. Kompfner, C. Lockwood, and G. Maycock, "Speed, speed limits and crashes," Tech. Rep. 58, TRL, Crowthorne, United Kingdom, 1994.

[10] C. Kloeden, G. Ponte, and A. McLean, "Travelling speed and the risk of crash involvement on rural roads," Tech. Rep. CR-204, Australian Transport Safety Bureau, 2001.

[11] O. Carsten and F. Tate, "External vehicle speed control," final report: integration, ITS, Univ. of Leeds, Leeds, 2000.

[12] J. Ehrlich, F. Saad, S. Lassarre, and S. Romon, "Assessment of LAVIA speed adaptation systems: experimental design and initial results on system use and speed behaviour," Proc. of 13th ITS World Congress, United Kingdom, 2006.

[13] A. Varhelyi and T. Makinen, "The effects of in-car speed limiters : Field studies," Transportation Research Part C: Emerging Technologies, vol.9(3), pp.191-211, 2001

[14] O. Carsten, F. Lai, K. Chorlton, P. Goodman, D. Carslaw, and S. Hess, "Speed limit adherence and its effect on road safety and climate change," tech. rep., University of Leeds - Institute for Transport Studies, october 2008.

[15] R. Driscoll, Y. Page, S. Lassarre, and J. Ehrlich, "LAVIA - an evaluation of the potential safety benefits of the french intelligent speed adaptation project," Annu. Proc. Assoc. Adv. Automot. Med., vol.51, pp.485-505, 2007.

[16] N. Barnes, A. Zelinsky, and L. Fletcher, "Real-time speed sign detection using the radial symmetry detector," IEEE Trans. Intell. Transp. Syst., vol.9(2), pp.322-332, 2008

[17] M. Ditze, F. Golatowski, N. Laum, A. Varhelyi, S. Gustafsson, and K. Geramani, "A survey on intelligen vehicle safety systems for adverse weather conditions," FISITA World Congress, 2010.

[18] C. Sentouh, S. Glaser, and S. Mammar, "Advanced vehicleinfrastructure-driver speed profile for road departure accident prevention," Vehicle System Dynamics, vol.44, pp.612-623, 2006.

[19] E. Bertolazzi, F. Biral, M. Da Lio, A. Saroldi, and F. Tango, "Supporting drivers in keeping safe speed and safe distance: The saspence subproject within the european framework programme 6 integrating project PReVENT," IEEE Transactions on Intelligent Transportation Systems, vol.11, no.3, pp.525-538, September 2010.

[20] A. Varhelyi, "Dynamic speed adaptation in adverse conditions -a system proposal-," IATSS Res., vol.26(2), pp.52-59, 2002.

[21] F. Jimenez, F. Aparicio, and J. Paez, "Evaluation of in-vehicle dynamic speed assistance in spain: algorithm and driver behaviour," IET Intell. Transp. Syst., vol.2(2), pp.132-142, 2008

[22] N. Hautière, J.P. Tarel, J. Lavenant, and D. Aubert, "Automatic fog detection and estimation of visibility distance through use of an onboard camera," Machine Vision and Applications Journal, vol.17(1), pp.8-20, 2006.

[23] R. Gallen, A. Cord, N. Hautière, and D. Aubert, "Towards night fog detection through use of in-vehicle multipurpose cameras," IEEE Intelligent Vehicles Symposium (IV'11), Baden Baden, Germany, 2011.

[24] A. Cord and D. Aubert, "Towards rain detection through use of invehicle multipurpose cameras," IEEE Intelligent Vehicles Symposium (IV'11), Baden Baden, Germany, 2011.

[25] J. Halimeh and M. Roser, "Raindrop detection on car windshields using geometric-photometric environment construction and intensitybased correlation," Proc. IEEE Intelligent Vehicles Symposium (IV’09), 2009.

[26] T. Teshima, H. Saito, M. Shimizu, and A. Taguchi, "Classification of wet/dry area based on the mahalanobis distance of feature from time space image analysis," Proc. IAPR on Machine Vision Applications, 2009.

[27] E. Romano, T. Kelley-Baker, and R. Voas, "Female involvement in fatal crashes: Increasingly riskier or increasingly exposed?" Accident Analysis and Prevention, vol.40, pp.1781-1788, 2008.

[28] L. Evans and P. Gerrish, "Antilock brakes and risk of front and rear impact in two-vehicle crashes," Accident Analysis and Prevention, vol.28(3), pp.315-323, 1996.

[29] D. Gabauer and H. Gabler, "Comparison of roadside crash injury metrics using event data recorders," Accident Analysis and Prevention, vol.40, pp.548-558, 2008. 
[30] D. Buzeman, D. Viano, and P. Lovsund, "Injury probability and risk in frontal crashes: Effects of sorting techniques on priorities for offset testing," Accident Analysis and Prevention, vol.30(5), pp.583-595, 1998.

[31] E. Miltner and H. Salwender, "Influencing factors on the injury severity of restrained front seat occupants in car-to-car head-on collisions," Accident Analysis and Prevention, vol.27(2), pp.143-150, 1995.

[32] S. Acierno, R. Kaufmanc, F. Rivara, and D. Grossman, "Vehicle mismatch: injury patterns and severity," Accident Analysis and Prevention, vol.36, pp.761-772, 2004.

[33] P. Mills and C. Hobbs, "The probability of injury to car occupants in frontal and side impacts," Stapp car crash conference, Chicago, IL, pp.223-232, 1984

[34] D. Gabauer and H. Gabler, "Comparison of the delta-v and occupant impact velocity crash severity metrics using event data recorders," Proceedings of the 50th Annual Association for the Advancement of Automotive Medicine Conference, Chicago, IL, USA, 2006.

[35] R. Ross, J. Lenard, B. Hurley, P. Thomas, D. Otte, and G. Vallet, "Crash severity calculations - theory and practice." STandardisation of Accident and Injury Registration Systems (STAIRS), 1998.

[36] D. Wood, N. Veyrat, C. Simms, and C. Glynn, "Limits for survivability in frontal collisions: Theory and real-life data combined," Accident Analysis and Prevention, vol.39, pp.679-687, 2007.

[37] D. Richards and C. Cuerden, "The relationship between speed and car driver injury severity," tech. rep., Transport Research Laboratory, London, UK, 2009.

[38] L. Patte, "Evaluation du risque en relation avec la visibilité," Proc. Prévention des risques et aides la conduite, 2010

[39] A. Aly, E.S. Zeidan, A. Hamed, and F. Salem, "An antilock-braking systems (ABS) control: A technical review," Intelligent Control and Automation, vol.2, pp.186-195, 2011.

[40] G. Louah, "The accuracy of a speed profile estimation method combining continuous and spot speed measurements," 13th Int. Conf. Road Safety on Four Continents, Warsaw, Poland, 2005.

[41] S. Gormer, A. Kummert, S.B. Park, and P. Egbert, "Vision-based rain sensing with an in-vehicle camera," Proc. IEEE Intelligent Vehicles Symposium (IV'09), 2009.

[42] M. Kane and M.T. Do, "A contribution of elastohydrodynamic lubrification for estimation of tire-road friction in wet conditions," International Conference on Tribology, Parma, Italy, September 2006

[43] N. Hautière and P. Lepert, "Dialogue between infrastructure and vehicles to improve road safety: The divas approach," Transport Research Arena (TRA'08), Ljubljana, Slovenia, 2008

[44] S. Glaser and V. Aguilera, "Vehicle-infrastructure-driver speed profile: towards the next generation of curve warning systems," Proc. 10th World Congress and Exhibition on Intelligent Transport Systems and Services, Madrid, Spain, 2003.

[45] P. Charbonnier and J.P. Tarel, "On the diagnostic of road pathway visibility," Proceedings of Transport Research Arena (TRA'10), Brussels, Belgium, 2010.

[46] S. Bordel, C. Charon, N. Hautière, and A. Somat, "Acceptabilité d'un système embarqué," 54e congrès de la Société Francaise de Psychologie, Montpellier, France, 2012.

[47] J. Nielsen, "Usability engineering. boston: Academic press," 1993.

[48] V. Venkatesh, M. Morris, G. Davis, and D. F.D., "User acceptance of information technology: Toward a unified view," MIS Quarterly: Management Information Systems, vol.27, pp.425-478, 2003.

[49] J. Blum and A. Eskandarian, "Managing effectiveness and acceptability in intelligent speed adaptation systems," Proc. IEEE Intelligent Transportation Systems Conference (ITSC'06), Toronto, Canada, 2006.

[50] E. Rendon-Velez, I. Horvath, and E. Opiyo, "Progress with situatio assessment and risk prediction in advanced driver assistance systems: a survey," ITS World Congress, Stockholm, Sweden, 2009.

[51] F. Jimenez, Y. Liang, and F. Aparicio, "Adapting ISA system warnings to enhance user acceptance," Accident Analysis and Prevention, 2010.

[52] J. Daniel, G. Pouly, A. Birouche, J.P. Lauffenburger, and M. Basset, "Navigation-based speed profile generation for an open road speed assistant," 12th IFAC Symposium on Control in Transportation Systems, Redondo Beach, CA, USA, 2009.

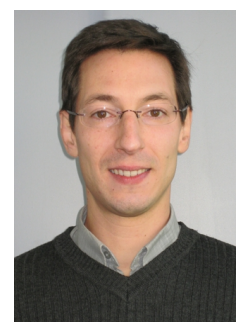

Romain Gallen received the M.S. degree in Computer Vision for Industrial Applications from the University Pierre et Marie Curie (UPMC), Paris 6, France and the Ph.D. degree respectively in 2005 and 2010. From 2005 to 2007, he was an engineer at the Laboratory on Interaction between Vehicle Infrastructure and Driver (LIVIC), which is a research unit from IFSTTAR, France. From 2007 to 2010, he was a PhD student on Visibility Risk Assessment in Adverse Weather Conditions using Computer Vision at both LIVIC and LEPSiS (Laboratory for road Operation, Perception, Simulations and Simulators), IFSTTAR France. Since 2011, he is head of the Information Processing Unit at CETMEF, France, and deals with maritime transport safety.

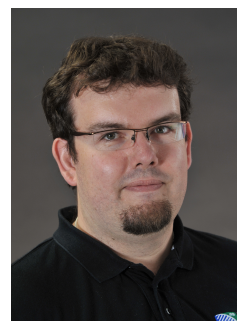

Nicolas Hautière received the M.S. degree in civil engineering from the National School of State Public Works (ENTPE), Vaulx en Velin, France in 2002 and the M.S. and Ph.D. degrees in computer vision from the University Jean Monnet, Saint-Etienne, France, in 2002 and 2005, respectively. He received the habilitation to manage research, HDR, in 2011 from the Université Paris-Est and the Specialized Master in political science and sustainable developement in 2013 from Ecole des Ponts ParisTech. Since 2009, he has been a research leader at the Laboratory for road Operation, Perception, Simulations and Simulators (LEPSiS). His research interests cover the modeling of the meteorological phenomena reducing the highway visibility, the detection of visibility conditions and the estimation of the visibility range.

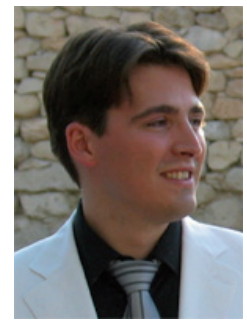

Aurélien Cord is a researcher in computer vision applied to intelligent transportation systems. He achieved his Ph.D. in 2003 on image processing for planetary surfaces, at Toulouse University. During 2004, he was working on content based image retrieval at Heudiasyc, Compiègne University. During 2005, he was working on the photometrical and spectral analysis of images from Mars-Express European mission, at the European Space Agency (ESA). From 2006 to 2007, he was working on automatic characterization of textured images for defect detection on steel plate images at the Centre de Morphologie Mathématique (CMM) of Mines ParisTech. Since 2008, he is a researcher at LIVIC, IFSTTAR. His research interests include analysis and interpretation of images and video from onboard cameras.

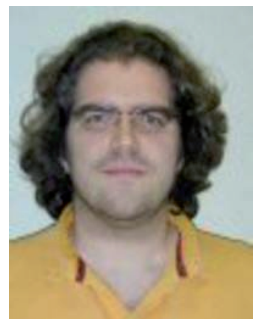

Sébastien Glaser received the Dipl.-Ing. degree from the National School of State Public Works (ENTPE), Vaulx en Velin, France, in 2000, the master's science degree in image analysis and synthesis from the Université Jean Monnet, Saint Etienne, France, the Ph.D. degree in automatic control, with emphasis on the vehicle dynamic analysis, from the Université d'Evry, Evry, France, in 2004, and the habilitation to manage research, HDR, in 2010. In 2004, he has been a Researcher with the Laboratoire sur les Interactions Véhicule Infrastructure Conducteur, which is a research unit of IFSTTAR, Versailles, France. He works on driving-assistance design and on driver studies. Since 2009, he has leaded a research team on Risk analysis, Decision making and Control. He is currently involved in several European Union initiatives (eFuture, HAVEit) and leads a French initiative on low-speed automation (ANR-ABV) 